\title{
Solutions of a Class of Sixth Order Boundary Value Problems Using the Reproducing Kernel Space
}

\author{
Ghazala Akram and Hamood Ur Rehman \\ Department of Mathematics, University of the Punjab, Lahore 54590, Pakistan \\ Correspondence should be addressed to Hamood Ur Rehman; hamood84@gmail.com
}

Received 25 October 2012; Revised 15 January 2013; Accepted 31 January 2013

Academic Editor: Lucas Jódar

Copyright ( 2013 G. Akram and H. Ur Rehman. This is an open access article distributed under the Creative Commons Attribution License, which permits unrestricted use, distribution, and reproduction in any medium, provided the original work is properly cited.

\begin{abstract}
The approximate solution to a class of sixth order boundary value problems is obtained using the reproducing kernel space method. The numerical procedure is applied on linear and nonlinear boundary value problems. The approach provides the solution in terms of a convergent series with easily computable components. The present method is simple from the computational point of view, resulting in speed and accuracy significant improvements in scientific and engineering applications.It was observed that the errors in absolute values are better than compared (Che Hussin and Kiliçman (2011) and, Noor and Mahyud-Din (2008), Wazwaz (2001), Pandey (2012)). Furthermore, the nonlinear boundary value problem for the integrodifferential equation has been investigated arising in chemical engineering, underground water flow and population dynamics, and other fields of physics and mathematical chemistry. The performance of reproducing kernel functions is shown to be very encouraging by experimental results.
\end{abstract}

\section{Introduction}

Boundary value problems arise in engineering, applied mathematics, and several branches of physics and have attracted much attention. However, it is difficult to obtain closed-form solutions for boundary value problems, especially for nonlinear problems. In most cases, only approximate solutions (either numerical solutions or analytical solutions) can be expected.

It is well known that a wide class of boundary value problems arise in various branches of pure and applied sciences including astrophysics, structural engineering, optimization, and economics. The literature of the numerical solution of sixth order boundary value problems is sparse. These types of problems generally arise in astrophysics; the narrow convecting layers bounded by stable layers which are believed to surround A-type stars may be modeled by sixth order boundary value problems [1].

Chandrasekhar [2] determined that when an infinite horizontal layer of fluid is heated from below and is under the action of rotation, instability sets in. When this instability is an ordinary convection, the ordinary differential equation is a sixth order. Details of the theorems which listed the conditions for the existence and uniqueness of solutions of sixth order boundary value problems are given in Agarwal [3], but no numerical methods are contained therein.

Higher order boundary value problems arise in the study of astrophysics, hydrodynamic and hydromagnetic stability, fluid dynamics, astronomy, beam and long wave theory, engineering, and applied physics. The boundary value problems of higher order have been investigated due to their mathematical importance and the potential for applications in diversified applied sciences.

Siddiqi and Akram $[4,5]$ presented a second order method using polynomial and nonpolynomial septic splines for the solution of linear sixth order boundary value problems with boundary conditions at first and second order derivatives. Noor and Mohyud-Din [6] proposed a reliable approach for solving linear and nonlinear sixth order boundary value problems by homotopy perturbation method. Wazwaz [7] used decomposition and modified domain decomposition methods to investigate the solution of sixth order nonlinear boundary value problems by making a comparison among differential transformation method (DTM) and Adomian decomposition method (ADM) [8]. Simos [9] proposed a new closed Newton-Cotes trigonometrically 
fitted differential method of high algebraic order. Simos [10] used a hybrid two-step method for the numerical solution of the radial Schrdinger equation and related problems with periodic or oscillating solutions. Anastassi and Simos [11] developed an efficient parametric symmetric linear phase fitted four-step method for the numerical solution of the Schrdinger equation and related oscillatory problems. A reproducing kernel Hilbert space is a useful framework for constructing approximate solutions of boundary value problems [12-15]. In this paper, a reproducing kernel method is used for the solution of a class of sixth order boundary value problems. To the best of our knowledge, the sixth order nonlinear boundary value problem for the integrodifferential equation which arises in chemical engineering, underground water flow and population dynamics, and other fields of physics and mathematical chemistry has not been investigated yet. It is illustrated that our methods can also be used to find the approximate solution of such integrodifferential equations.

Consider the following sixth order two-point boundary value problem (BVP):

$$
\begin{gathered}
u^{(6)}(x)+\sum_{i=0}^{2} a_{i}(x) u^{(2 i)}(x)+\int_{0}^{x} g(x, s)(u(s))^{2} d s \\
=f(x, u(x)), \quad 0 \leq x \leq 1, \\
u^{(i)}(0)=A_{i}, \quad u^{(i)}(1)=B_{i}, \quad i=0,1,2,
\end{gathered}
$$

where $A_{i}$ and $B_{i}, i=0,1,2$, are finite real constants and $a_{i}(x), i=0,1,2$, and $f(x, u(x))$ are the continuous functions on $[0,1]$. Let the differential operator be $L$, and homogenization of the boundary conditions of (1) can be transformed into the following form:

$$
\begin{gathered}
L u=f(x, u(x)), \quad a \leq x \leq b, \\
u^{(i)}(0)=0, \quad u^{(i)}(1)=0, \quad i=0,1,2 .
\end{gathered}
$$

Therefore, to solve (1), it suffices to solve (2).

The rest of this paper is organized as follows. In Section 2, the reproducing kernel function is obtained, and reproducing kernel spaces needed in this paper are defined. After that in Section 3, a solution of (2) with initial boundary value conditions is presented. Finally, numerical examples are discussed to demonstrate the accuracy of the presented method in Section 4.

\section{Reproducing Kernel Spaces}

(i) The reproducing kernel space $W_{2}^{7}[0,1]$ is defined by $W_{2}^{7}[0,1]=\left\{u(x) \mid u^{(i)}(x), i=0,1, \ldots, 6\right.$, are absolutely continuous real-valued functions in $[0,1]$, $\left.u^{(7)} \in L^{2}[0,1]\right\}$. The inner product in $W_{2}^{7}[0,1]$ is given by

$$
\begin{array}{r}
\langle u(x), v(x)\rangle=\int_{0}^{1}\left(u^{(6)}(x) v^{(6)}(x)+u^{(7)}(x) v^{(7)}(x)\right) d x \\
u(x), v(x) \in W_{2}^{7}[0,1] .
\end{array}
$$

(ii) The reproducing kernel space $W_{2}^{1}[0,1]$ is defined by $W_{2}^{1}[0,1]=\{u(x) \mid u(x)$ is absolutely continuous realvalued function in $\left.[0,1], u^{(1)} \in L^{2}[0,1]\right\}$. Also, the inner product is given by

$$
\begin{array}{r}
\langle u(x), v(x)\rangle=\int_{0}^{1}\left(u(x) v(x)+u^{(1)}(x) v^{(1)}(x)\right) d x, \\
u(x), v(x) \in W_{2}^{1}[0,1] .
\end{array}
$$

Theorem 1. The space $W_{2}^{7}[0,1]$ is a reproducing kernel Hilbert space. That is, for all $u(y) \in W_{2}^{7}[0,1]$ and each fixed $x \in$ $[0,1], y \in[0,1]$, there exists $R_{x}(y) \in W_{2}^{7}[0,1]$ such that $\left\langle u(y), R_{x}(y)\right\rangle=u(x)$, and $R_{x}(y)$ is called the reproducing kernel function of space $W_{2}^{7}[0,1]$. The reproducing kernel function $R_{x}(y)$ in $[0,1]$ is given by

$$
R_{x}(y)= \begin{cases}\sum_{i=0}^{11} c_{i} y^{i}+c_{12} e^{y}+c_{13} e^{-y}, & y \leq x . \\ \sum_{i=0}^{11} d_{i} y^{i}+d_{12} e^{y}+d_{13} e^{-y}, & y>x .\end{cases}
$$

The coefficients $c_{i}, d_{i}(i=0,1,2, \ldots, 13)$ are given in the appendix at the end.

\section{The Exact and Approximate Solutions}

The solution of (2) is given in the reproducing kernel Hilbert space $W_{2}^{7}[0,1]$, and the linear operator $L: W_{2}^{7}[0,1] \rightarrow$ $W_{2}^{1}[0,1]$ is bounded. Choose a countable dense subset $D=$ $\left\{x_{i}\right\}_{i=1}^{\infty}$ in the domain $[0,1]$, and let

$$
\varphi_{i}(x)=Q_{x_{i}}(y), \quad i \in N
$$

where $Q_{x_{i}}(y) \in W_{2}^{1}[0,1]$ is reproducing kernel of $W_{2}^{1}[0,1]$. Further assume that $\psi_{i}(x)=\left(L^{*} \varphi_{i}\right)(x)$, where $L^{*}$ : $W_{2}^{1}[0,1] \rightarrow W_{2}^{7}[0,1]$ is the conjugate operator of $L$.

Theorem 2. $\left\{\psi_{i}(x)\right\}_{i=1}^{\infty}$ is a complete system of $W_{2}^{7}[0,1]$ and $\psi_{i}(x)=\left.L_{y} R_{x}(y)\right|_{y=x_{i}}$.

Proof. For each fixed $u(x) \in W_{2}^{7}[0,1]$, let $\left\langle u(x), \psi_{i}(x)\right\rangle=0$ $(i=1,2, \ldots)$, which implies that

$$
\left\langle u(x),\left(L^{*} \varphi_{i}\right)(x)\right\rangle=\left\langle L u(x), \varphi_{i}(x)\right\rangle=(L u)\left(x_{i}\right)=0 .
$$


Since $\left\{x_{i}\right\}_{i=1}^{\infty}$ is dense in $[0,1],(L u)(x)=0$, which implies that $u \equiv 0$ from the existence of $L^{-1}$.

Using reproducing kernel property, it can be written as

$$
\begin{aligned}
\psi_{i}(x) & =\left\langle\psi_{i}(y), R_{x}(y)\right\rangle \\
& =\left\langle\left(L^{*} \varphi_{i}\right)(x), R_{x}(y)\right\rangle \\
& =\left\langle\varphi_{i}(y), L R_{x}(y)\right\rangle \\
& =\left.L_{y} R_{x}(y)\right|_{y=x_{i}} .
\end{aligned}
$$

To orthonormalize the sequence $\left\{\psi_{i}\right\}_{i=1}^{\infty}$ in the reproducing kernel space $W_{2}^{7}[0,1]$, Gram-Schmidt process can be used as

$$
\bar{\psi}_{i}(x)=\sum_{k=1}^{i} \beta_{i k} \psi_{k}(x), \quad i=1,2, \ldots
$$

Theorem 3. If $\left\{x_{i}\right\}_{i=1}^{\infty}$ is dense in $[0,1]$ and the solution of (2) is unique, for all $u(x) \in W_{2}^{7}[0,1]$, the series is convergent in the norm of $\|\cdot\|_{W_{2}^{7}}$. If $u(x)$ is exact solution; then the solution of (2) has the form

$$
u(x)=\sum_{i=1}^{\infty} \sum_{k=1}^{i} \beta_{i k} f\left(x_{k}, u\left(x_{k}\right)\right) \bar{\psi}_{i}(x) .
$$

Proof. Since $u(x) \in W_{2}^{7}[0,1]$ and can be expanded in the form of Fourier series about normal orthogonal system $\left\{\psi_{i}\right\}_{i=1}^{\infty}$ as

$$
u(x)=\sum_{i=1}^{\infty}\left\langle u(x), \bar{\psi}_{i}(x)\right\rangle \bar{\psi}_{i}(x) .
$$

Since the space $W_{2}^{7}[0,1]$ is Hilbert space, so the series $\sum_{i=1}^{\infty}\left\langle u(x), \bar{\psi}_{i}(x)\right\rangle \bar{\psi}_{i}(x)$ is convergent in the norm of $\|\cdot\|_{W_{2}^{7}}$. From (9) and (11), it can be written as

$$
\begin{aligned}
u(x) & =\sum_{i=1}^{\infty}\left\langle u(x), \bar{\psi}_{i}(x)\right\rangle \bar{\psi}_{i}(x) \\
& =\sum_{i=1}^{\infty}\left\langle u(x), \sum_{k=1}^{i} \beta_{i k} \psi_{k}(x)\right\rangle \bar{\psi}_{i}(x) \\
& =\sum_{i=1}^{\infty} \sum_{k=1}^{i} \beta_{i k}\left\langle u(x), \psi_{k}(x)\right\rangle \bar{\psi}_{i}(x) \\
& =\sum_{i=1}^{\infty} \sum_{k=1}^{i} \beta_{i k}\left\langle u(x),\left(L^{*} \varphi_{k}\right)(x)\right\rangle \bar{\psi}_{i}(x) \\
& =\sum_{i=1}^{\infty} \sum_{k=1}^{i} \beta_{i k}\left\langle L u(x), \varphi_{k}(x)\right\rangle \bar{\psi}_{i}(x) .
\end{aligned}
$$

If $u(x)$ is the exact solution of (2) and $L u=f(x, u(x))$, then

$$
\begin{aligned}
u(x) & =\sum_{i=1}^{\infty} \sum_{k=1}^{i} \beta_{i k}\left\langle f(x, u(x)), \varphi_{k}(x)\right\rangle \bar{\psi}_{i}(x) \\
& =\sum_{i=1}^{\infty} \sum_{k=1}^{i} \beta_{i k} f\left(x_{k}, u\left(x_{k}\right)\right) \bar{\psi}_{i}(x) .
\end{aligned}
$$

The approximate solution of $u(x)$ is given by

$$
u_{n}(x)=\sum_{i=1}^{n} \sum_{k=1}^{i} \beta_{i k} f\left(x_{k}, u\left(x_{k}\right)\right) \bar{\psi}_{i}(x) .
$$

Remark 4. If (2) is linear, that is, $f(x, u(x))=f(x)$, then the solution can be obtained directly from (14).

If (2) is nonlinear, the approximate solution can be obtained using the following method.

Using (13), the following iterative formula can be constructed as

$$
\begin{aligned}
& u_{0}(x)=0, \\
& u_{n+1}=\sum_{i=1}^{\infty} \sum_{k=1}^{i} \beta_{i k} f\left(x_{k}, u_{n}\left(x_{k}\right)\right) \bar{\psi}_{i}(x) .
\end{aligned}
$$

It can be noted that the approximate solution $u_{n}(x)$ given in (15) satisfied the boundary conditions of problem (2). In fact, the solution of problem (2) is considered as the fixed point of the following functional under the suitable choice of the initial term $u_{0}(x)$ :

$$
u_{n+1}=\sum_{i=1}^{\infty} \sum_{k=1}^{i} \beta_{i k} f\left(x_{k}, u_{n}\left(x_{k}\right)\right) \bar{\psi}_{i}(x) .
$$

Theorem 5 (Banach's fixed point theorem). Assume that $X$ is a Banach space and $A: X \rightarrow X$ is a nonlinear function, and assume that

$$
\|A[u]-A[v]\| \leq \alpha\|u-v\|, \quad u, v \in X,
$$

for some constants $\alpha<1$. Then, A has a unique fixed point. Furthermore, the sequence $u_{n+1}=A\left[u_{n}\right]$ with an arbitrary choice of $u_{0} \in X$ converges to the fixed point of $A$.

According to Theorem 5, for the nonlinear mapping,

$$
A[u(x)]=\sum_{i=1}^{\infty} \sum_{k=1}^{i} \beta_{i k} f\left(x_{k}, u_{n}\left(x_{k}\right)\right) \bar{\psi}_{i}(x) .
$$

a sufficient condition for convergence of the present iteration method is strictly contraction of A. Furthermore, the sequence in (15) converges to the fixed point of A which is also the solution of problem (2).

The approximate solution $u_{n, m}(x)$ can be obtained by taking finitely many terms in the series representation of $u_{n}(x)$, given by

$$
u_{n, m}(x)=\sum_{i=1}^{n} \sum_{k=1}^{\mathrm{i}} \beta_{i k} f\left(x_{k}, u_{m-1}\left(x_{k}\right)\right) \bar{\psi}_{i}(x) .
$$


TABLE 1: Maximum absolute error for problem (20) $\left(\operatorname{Max}\left|u-u_{20,1}\right|\right.$ with maximum CPU time $(s)=0.315$ for all cases of $\left.c\right)$.

\begin{tabular}{lcccc}
\hline$c=1$ & $c=10$ & $c=100$ & $c=1000$ & $c=100000$ \\
\hline $6.1746 E-10$ & $3.05092 E-9$ & $1.16991 E-8$ & $1.51919 E-9$ & $1.07433 E-8$ \\
\hline
\end{tabular}

TABLE 2: Absolute error obtained by the present method and other methods [8] for problem (22) with CPU time $(s)=0.331$.

\begin{tabular}{lccc}
\hline$x$ & Present method $(n=20, m=1)$ & Wazwaz [7] & Noor and Mohyud-Din [6] \\
\hline 0.0 & 0 & 0 & 0 \\
0.1 & 0 & $1.233 E-4$ & $1.233 E-4$ \\
0.2 & $2.66 E-15$ & $2.354 E-4$ & $2.354 E-4$ \\
0.3 & $6.66 E-15$ & $3.257 E-4$ & $3.257 E-4$ \\
0.4 & $9.10 E-15$ & $3.855 E-4$ & $3.855 E-4$ \\
0.5 & $1.08 E-14$ & $4.086 E-4$ & $4.086 E-4$ \\
0.6 & $1.04 E-14$ & $3.919 E-4$ & $3.919 E-4$ \\
0.7 & $8.43 E-15$ & $3.361 E-4$ & $3.361 E-4$ \\
0.8 & $3.55 E-15$ & $2.459 E-4$ & $2.459 E-4$ \\
0.9 & $4.44 E-16$ & $1.299 E-4$ & $1.299 E-4$ \\
1.0 & 0 & $2.000 E-9$ & $2.000 E-9$ \\
\hline
\end{tabular}

\section{Numerical Examples}

To illustrate the applicability and effectiveness of our method, three numerical examples are constructed. All the numerical computations are performed by using Mathematica 5.2 on a system with Intel Core 2 Quad $2.6 \mathrm{GHz} \mathrm{CPU}$ and $3 \mathrm{~GB}$ of RAM.

Example 6. Consider the following special sixth order boundary value problem involving a parameter $c[6]$ :

$$
\begin{gathered}
u^{(6)}(x)=(1+c) u^{(4)}(x) \\
-c u^{(2)}(x)+c x, \quad 0 \leq x \leq 1, \\
u(0)=u^{(1)}(0)=1, u^{(2)}(0)=0, \\
u(0)=\frac{7}{6}+\sinh 1, \quad u^{(1)}(1)=\frac{1}{2}+\cosh 1, \\
u^{2}(1)=1+\sinh 1 .
\end{gathered}
$$

The exact solution of problem (20) is

$$
u(x)=1+\frac{1}{6} x^{3}+\sinh x
$$

A comparison of the errors in absolute values between the method developed in this paper and that of Noor and Mohyud-Din [6] is shown in Table 1. For small values of $c=1,10,1000$, and 1000, it is noted that the results obtained by our method are better than the method developed by Noor and Mohyud-Din [6]. The results obtained by Noor and Mohyud-Din using Adomian's decomposition method, variation iteration method, and Homotopy perturbation method for large values of $c=1000000$ are no longer valid as given in [6], but the results obtained by the present method for Example 6 are also valid and accurate for large value of $c=1000000$.

Example 7. Consider the following nonlinear boundary value problem of sixth order $[6,7]$ :

$$
\begin{gathered}
u^{(6)}(x)=e^{-x}(u(x))^{2}, \quad 0 \leq x \leq 1, \\
u(0)=u^{(1)}(0)=u^{(2)}(0)=1 \\
u(1)=u^{(1)}(1)=u^{(2)}(1)=e .
\end{gathered}
$$

The exact solution of problem (22) is

$$
u(x)=e^{x}
$$

A comparison of the errors in absolute values between the method developed in this paper and that of Wazwaz [7] and Noor and Mohyud-Din [6] is shown in Table 2 and Figures 1 and 2.

Example 8. Consider the following nonlinear boundary value problem of sixth order:

$$
\begin{gathered}
u^{(6)}(x)=e^{x}(u(x))^{3}, \quad 0<x<1, \\
u(0)=1, \quad u^{(1)}(0)=\frac{-1}{2}, \quad u^{(2)}(0)=\frac{1}{4}, \\
u(1)=e^{-1 / 2}, \quad u^{(1)}(1)=\frac{-1}{2} e^{-1 / 2}, \quad u^{(2)}(1)=\frac{1}{4} e^{-1 / 2} .
\end{gathered}
$$

The exact solution of problem (24) is

$$
u(x)=e^{-x / 2} .
$$


TABLE 3: Comparison of the numerical results for problem (24) with CPU time $(s)=0.379$.

\begin{tabular}{lcccc}
\hline$x$ & Exact & DTM $[8]$ & ADM [8] & Present method $u_{20,1}$ \\
\hline 0.1 & 1 & 1 & 1 & 1 \\
0.1 & 0.9512294245 & 0.9492075127 & 0.9492075127 & 0.9512294245 \\
0.2 & 0.9048374181 & 0.8916268943 & 0.8916268943 & 0.9048374180 \\
0.3 & 0.8607079765 & 0.8251427077 & 0.8251427077 & 0.8607079764 \\
0.4 & 0.8187307532 & 0.7534730280 & 0.7534730280 & 0.8187307531 \\
0.5 & 0.7788007831 & 0.6839803183 & 0.6839803183 & 0.7788007831 \\
0.6 & 0.7408182206 & 0.6254839642 & 0.6254839642 & 0.7408182207 \\
0.7 & 0.7046880897 & 0.7046783358 & 0.5860748693 & 0.7046880897 \\
0.8 & 0.6703200461 & 0.6703157625 & 0.5709325210 & 0.6703200460 \\
0.9 & 0.6376281517 & 0.6376273947 & 0.5801448253 & 0.6376281516 \\
1 & 0.6065306598 & .6065306599 & 0.6065306590 & 0.6065306597 \\
\hline
\end{tabular}

TABLE 4: Absolute and relative error between exact and approximate solutions for problem (26) with CPU time $(s)=2.359$.

\begin{tabular}{lcc}
\hline$x$ & $\begin{array}{c}\text { Absolute error } \\
(n=20, m=1)\end{array}$ & $\begin{array}{c}\text { Relative error } \\
(n=20, m=1)\end{array}$ \\
\hline 0.1 & $4.70 E-7$ & $4.69 E-6$ \\
0.2 & $2.73 E-6$ & $1.35 E-5$ \\
0.3 & $6.37 E-6$ & $2.09 E-5$ \\
0.4 & $9.82 E-6$ & $2.39 E-5$ \\
0.5 & $114 E-5$ & $2.19 E-5$ \\
0.6 & $104 E-5$ & $1.64 E-5$ \\
0.7 & $7.21 E-6$ & $9.51 E-6$ \\
0.8 & $3.28 E-6$ & $3.70 E-6$ \\
0.9 & $6.02 E-7$ & $5.86 E-7$ \\
1 & $4.44 E-16$ & $3.77 E-16$ \\
\hline
\end{tabular}

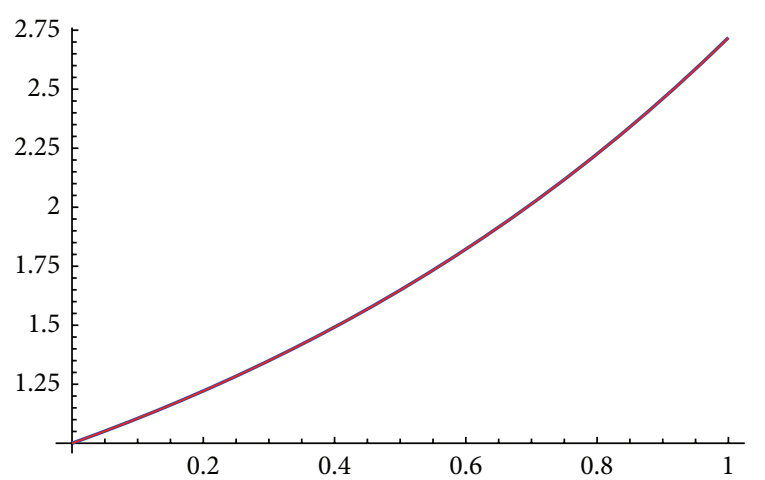

FIGURE 1: Blue line: exact solution. Red line: approximate solution.

A comparison of the errors in absolute values between the method developed in this paper and that of Che Hussin and Kiliçman [8] is shown in Table 3 and Figures 3 and 4.
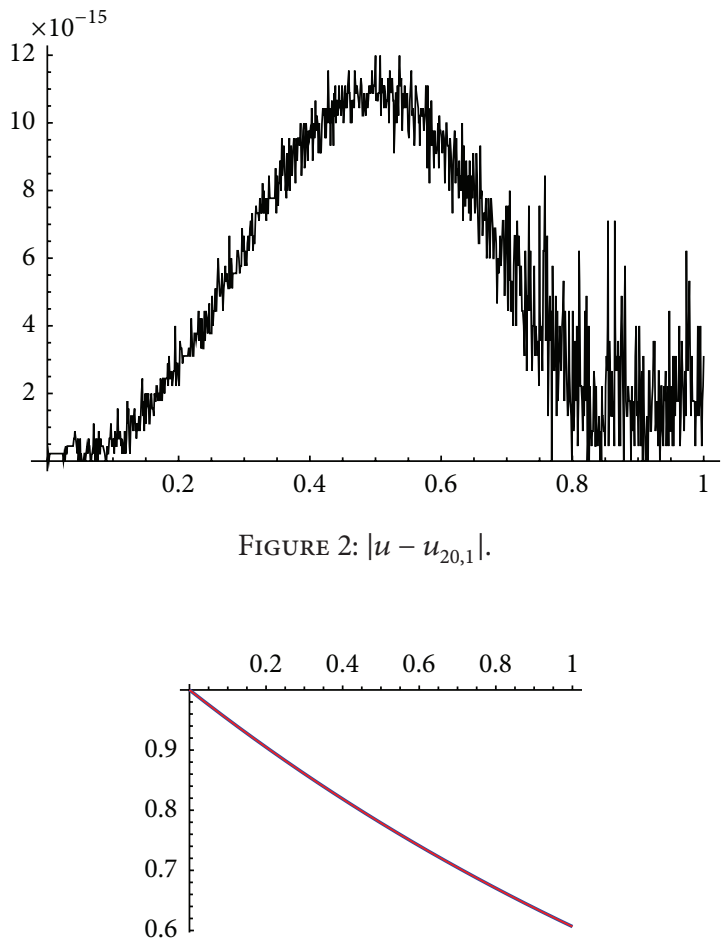

FIGURE 3: Blue line: exact solution. Red line: approximate solution.

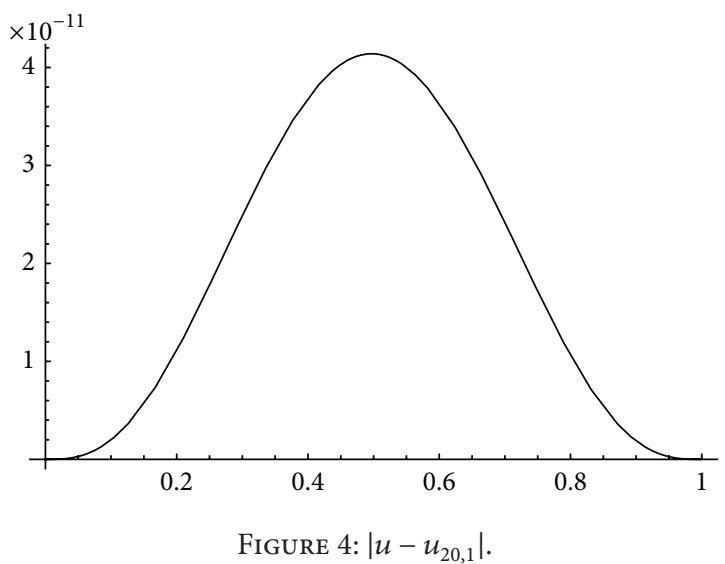


TABLE 5: Maximum absolute error for problem $(28)(m=1)$.

\begin{tabular}{lccccc}
\hline & $n=8$ & $n=16$ & $n=32$ & $n=64$ & $n=128$ \\
\hline MAE [16] & $.101414 E-2$ & $.569422 E-4$ & $.346105 E-5$ & $.205712 E-6$ & $.250685 E-9$ \\
MAE (present method) & $.4647 E-4$ & $.4728 E-5$ & $.38191 E-6$ & $.4982 E-8$ & $.2264 E-10$ \\
\hline
\end{tabular}

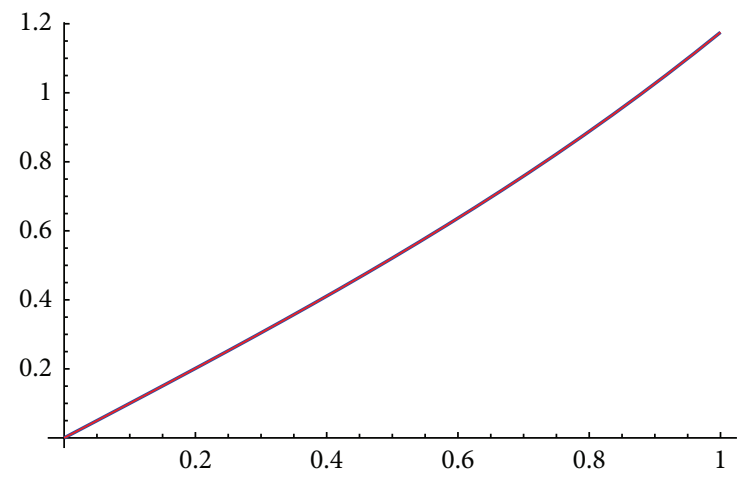

FIGURE 5: Blue line: exact solution. Red line: approximate solution.

Example 9. Consider the following nonlinear integral boundary value problem of sixth order:

$$
\begin{gathered}
u^{(6)}(x)+u^{(4)}(x)+\int_{0}^{x} e^{s}(u(s))^{2} d s=f(x), \quad 0<x<1, \\
u(0)=0, \quad u^{(1)}(0)=1, \quad u^{(2)}(0)=0, \\
u(1)=\sinh 1, \quad u^{(1)}(1)=\cosh 1, \quad u^{(2)}(1)=\sinh 1 .
\end{gathered}
$$

The exact solution of problem (24) is

$$
u(x)=\sinh x f(x)=2 \sinh x-\frac{1}{12} e^{-x}\left(e^{x}-1\right)^{3}\left(e^{x}+3\right) .
$$

The absolute error (between exact and approximate solutions) and relative error are given in Table 4 and Figures 5 and 6.

Example 10. Consider the following nonlinear boundary value problem of sixth order:

$$
\begin{array}{ll}
u^{(6)}(x)=u^{(1)}(x) u^{(5)}(x) & \\
+\left(u^{(3)}(x)\right)^{3}+f(x), & 0<x<1, \\
u(0)=0, & u^{(1)}(0)=\pi, \quad u^{(2)}(0)=0, \\
u(1)=0, & u^{(1)}(1)=-\pi, \quad u^{(2)}(1)=0 .
\end{array}
$$

The exact solution of problem (24) is

$$
u(x)=\sin \pi x .
$$

It is observed that the results obtained from our method which are obtained in the form of maximum absolute error

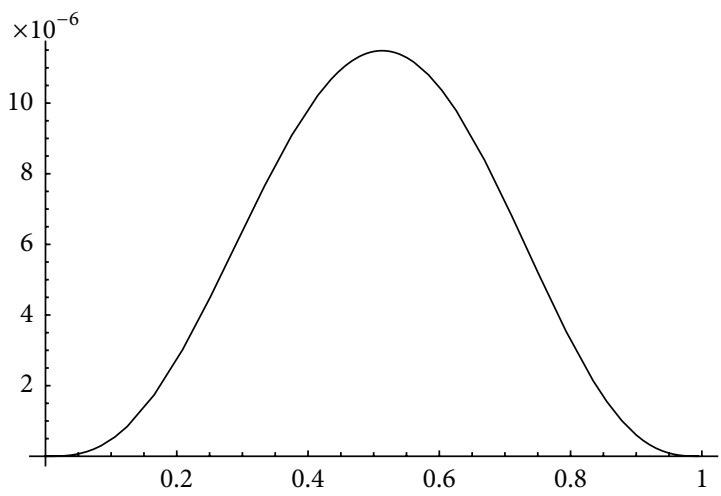

FigURE 6: $\left|u-u_{20,1}\right|$.

(MAE) are better than the results obtained from the finite difference method [16] as shown in Table 5. It is noted that CPU time (s) for $n=8$ is $0.346, n=16$ is $0.416, n=32$ is $0.687, n=64$ is 1.886 , and $n=128$ is 4.366 .

\section{Conclusion}

In this paper, a reproducing kernel space method to find the solution of a class of sixth order boundary value problems is considered. The properties of the reproducing kernel space require no more integral computation for some functions, instead of computing some values of a function at some nodes. This simplification of integral computation not only improves the computational speed but also improves the computational accuracy. It was observed that the errors in absolute values are better than the other developed methods $[6-8,16]$. Mathematica software is used for all computational work. The numerical results show that only a few number of iteration steps can be used for numerical purpose with a high degree of accuracy. It is analyzed that our proposed method is well suited for use in higher order boundary value problems as it provides the best solution in a less number of iterations and reduces the computational work. Therefore, the present method is an accurate and reliable analytical technique for sixth order boundary value problems.

\section{Appendix}

$$
\begin{aligned}
& c_{0}=e^{-x}\left(-2 e^{2}-2 e^{2}-e^{x}\left(-2 e x^{3}(21+x(-32+13 x))+\right.\right. \\
& (-1+x)^{3}(2+x(6+13 x))+e^{2}(-1+x)^{3}(2+x(6+ \\
& 13 x)))) / 2\left(-1+e^{2}\right), \\
& c_{1}=(-1+x)^{3}(2+x(6+13 x)) / 2,
\end{aligned}
$$


$c_{2}=e^{-x}\left(-2 e^{2}-2 e^{2 x}-e^{x}\left(-2 e x^{3}(21+x(-32+13 x))+\right.\right.$ $(-1+x)^{3}(2+x(6+13 x))+e^{2}(-1+x)^{3}(2+x(6+$ $13 x)))) / 4\left(-1+e^{2}\right)$,

$c_{3}=\left(1 /\left(39916800\left(-1+e^{2}\right)\right)\right)\left(e^{-x}\left(-19958400 e^{2}\right.\right.$ $x(-23+21 e)+19958400 e(-21+23 e)+$ $e^{x}(-13305600(34+7 e(-9+5 e))+459043200(-1+$ $\left.e^{2}\right) x-6652800(34+7 e(-9+5 e)) x^{2}+200(70169941+$ $e(-96399072+26628299 e)) x^{3}-2835(7328513+$ $e(-10060160+2745727 e)) x^{4}+13(-1+e)$ $(-638372743+239796857 e) x^{5}-341880(-1+$ $\left.e^{2}\right) x^{6}+91080\left(-1+e^{2}\right) x^{7}-6105\left(-1+e^{2}\right) x^{8}+$ $\left.\left.1265\left(-1+e^{2}\right) x^{9}-66\left(-1+e^{2}\right) x^{10}+10\left(-1+e^{2}\right) x^{11}\right)\right)$, $c_{4}=\left(1 /\left(39916800\left(-1+e^{2}\right)\right)\right)\left(e^{-} x\left(19958400 e^{2 x}(-33+\right.\right.$ $32 e)-19958400 e(-32+33 e)+e^{x}(19958400(33+$ $e(-64+33 e))-656964000\left(-1+e^{2}\right) x+9979200(33+$ $e(-64+33 e)) x^{2}-315(65957497+e(-90541440+$ $24710663 e)) x^{3}+80(385150973+e(-526901760+$ $142000267 e)) x^{4}-3(-1+e)(-4106240179+$ $1515375821 e) x^{5}+443520\left(-1+e^{2}\right) x^{6}-130350$ $\left(-1+e^{2}\right) x^{7}+7920\left(-1+e^{2}\right) x^{8}-1815\left(-1+e^{2}\right) x^{9}+$ $\left.\left.\left.88\left(-1+e^{2}\right) x^{10}-15\left(-1+e^{2}\right) x^{11}\right)\right)\right)$,

$c_{5}=(1 / 39916800(1+e))\left(e^{-x}(259459200 e-\right.$ $259459200 e^{2 x}+e^{x}(-332640(-779+781 e)+$ $259459200(1+e) x-166320(-779+781 e) x^{2}+$ $13(-638372743+239796857 e) x^{3}-3(-4106235559+$ $1515380441 e) x^{4}+42(-117317897+43299703 e) x^{5}-$ $166782(1+e) x^{6}+51480(1+e) x^{7}-2970(1+e) x^{8}+$ $\left.\left.\left.715(1+e) x^{9}-33(1+e) x^{10}+6(1+e) x^{11}\right)\right)\right)$,

$c_{6}=-(-1+x)^{3} x(1+3 x) / 720$,

$c_{7}=(-1+x)^{3}(2+x(6+13 x)) / 10080$,

$c_{8}=-(-1+x)^{3} x(1+3 x) / 40320$,

$c_{9}=(-1+x)^{3}(2+x(6+13 x)) / 725760$,

$c_{10}=-(-1+x)^{3} x(1+3 x) / 3628800$,

$c_{11}=(-1+x)^{3}\left(1+3 x+6 x^{2}\right) / 39916800$,

$c_{12}=e^{-x}\left(e^{2}+e^{2 x}+e^{1+x} x^{3}(21+(-32+13 x) x)+\right.$ $\left.\left.e(-1+x)^{3}(2+x(6+13 x))\right)\right) / 2\left(e^{2}-1\right)$,

$c_{13}=e^{-x}\left(e^{2}+e^{2 x}+e^{1+x}\left(x^{3}(-21+(32-13 x) x)+\right.\right.$ $\left.\left.e(-1+x)^{3}(2+x(6+13 x))\right)\right) / 2\left(e^{2}-1\right)$,

$d_{0}=39916800\left(-1+e^{2}\right)\left(e-x\left(-39916800 e^{2}-\right.\right.$ $39916800 e^{2 x}-e^{x}\left(-39916800\left(1+e^{2}\right)+39916800(-1+\right.$ $\left.e^{2}\right) x-19958400\left(1+e^{2}\right) x^{2}+13305600(34+7 e(-9+$ $5 e)) x^{3}-19958400(33+e(-64+33 e)) x^{4}+332640(-1+$ e) $(-779+781 e) x^{5}+7920\left(-1+e^{2}\right) x^{7}+110\left(-1+e^{2}\right) x^{9}+$ $\left.\left.\left.\left(-1+e^{2}\right) x^{11}\right)\right)\right)$,

$d_{1}=x^{3}(41731200+x(-59724000+x(23587200+$ $\left.\left.\left.5040 x+90 x^{3}+x^{5}\right)\right)\right) / 3628800$,

$d_{2}=\left(1 / 725760\left(-1+e^{2}\right)\right)\left(e^{-x}\left(-362880 e^{2}-362880 e^{2 x}\right.\right.$ $-e^{x}\left(-362880\left(1+e^{2}\right)+362880\left(-1+e^{2}\right) x-181440(1+\right.$ $\left.e^{2}\right) x^{2}+120960(34+7 e(-9+5 e)) x^{3}-181440(33+$ $e(-64+33 e)) x^{4}+3024(-1+e)(-779+781 e) x^{5}+72(-1+$ $\left.\left.\left.\left.e^{2}\right) x^{7}+\left(-1+e^{2}\right) x^{9}\right)\right)\right)$,

$d_{3}=\left(1 / 39916800\left(-1+e^{2}\right)\right)\left(e^{-x}\left(-19958400 e^{2 x}(-23+\right.\right.$ $21 e)+19958400 e(-21+23 e)+e^{x}(-19958400(23+$ $e(-42+23 e))+459043200\left(-1+e^{2}\right) x-9979200(23+$ $e(-42+23 e)) x^{2}+200(70169941+e(-96399072+$ $26628299 e)) x^{3}-315(65957497+e(-90541440+$ $24710663 e)) x^{4}+13(-1+e)(-638372743+$ $39796857 e) x^{5}-332640\left(-1+e^{2}\right) x^{6}+91080(-1+$ $\left.e^{2}\right) x^{7}-5940\left(-1+e^{2}\right) x^{8}+1265\left(-1+e^{2}\right) x^{9}-66(-1+$ $\left.\left.\left.\left.e^{2}\right) x^{10}+10\left(-1+e^{2}\right) x^{11}\right)\right)\right)$,

$d_{4}=\left(1 / 39916800\left(-1+e^{2}\right)\right)\left(e^{-x}\left(19958400 e^{2 x}(-33+\right.\right.$ $32 e)-19958400 e(-32+33 e)+e^{x}(19958400(33+$ $e(-64+33 e))-658627200\left(-1+e^{2}\right) x+9979200(33+$ $e(-64+33 e)) x^{2}-2835(7328513+e(-10060160+$ $2745727 e)) x^{3}+80(385150973+e(-526901760+$ $142000267 e)) x^{4}-3(-1+e)(-4106235559+$ $1515380441 e) x^{5}+443520\left(-1+e^{2}\right) x^{6}-130680(-1+$ $\left.e^{2}\right) x^{7}+7920\left(-1+e^{2}\right) x^{8}-1815\left(-1+e^{2}\right) x^{9}+88(-1+$ $\left.\left.\left.\left.e^{2}\right) x^{10}-15(-1+e) x^{11}\right)\right)\right)$,

$d_{5}=(1 / 39916800(1+e)) e^{-x}(259459200 e-$ $259459200 e^{2 x}+e^{x}(-259459200(-1+e)+$ $259459200(1+e) x-129729600(-1+e) x^{2}+$ $13(-638372743+239796857 e) x^{3}-3(-4106240179+$ $1515375821 e) x^{4}+42(-117317897+43299703 e) x^{5}-$ $166320(1+e) x^{6}+51480(1+e) x^{7}-2970(1+e) x^{8}+$ $\left.\left.715(1+e) x^{9}-33(1+e) x^{10}+6(1+e) x^{11}\right)\right)$,

$d_{6}=-x^{3}(740+x(-960+361 x)) / 86400$,

$d_{7}=x^{3}(276+x(-395+156 x)) / 120960$,

$d_{8}=-\left(x^{3}(37+6 x(-8+3 x))\right) / 241920$,

$d_{9}=-\left(x^{3}(23+x(-33+13 x))\right) / 725760$,

$d_{10}=-\left(x^{3}(6+x(-8+3 x))\right) / 39916800$,

$d_{11}=x^{3}(10+3 x(-5+2 x)) / 39916800$,

$d_{12}=\left(-2+x^{2}(-1+x(23+x(-33+13 x)+e(-21+\right.$ $(32-13 x) x)))+2 \cosh x) / 2\left(e^{2}-1\right)$,

$d_{13}=\left(e\left(x^{3}(-21+(32-13 x) x)+e(-1+x)^{3}(2+x(6+\right.\right.$ $13 x)))+2 e \cosh x)) / 2\left(e^{2}-1\right)$.

\section{References}

[1] J. Toomre, J. R. Zahn, J. Latour, and E. A. Spiegel, "Stellar convection theory ii:single mode study of the second convection zone in a-type stars," The Astrophysical Journal, vol. 207, pp. 545$563,1976$.

[2] S. Chandrasekhar, Hydrodynamic and Hydromagnetic Stability, The International Series of Monographs on Physics, Clarendon Press, Oxford, UK, 1961.

[3] R. P. Agarwal, Boundary Value Problems for Higher Order Differential Equations, World Scientific Publishing, Singapore, 1986.

[4] G. Akram and S. S. Siddiqi, "Solution of sixth order boundary value problems using non-polynomial spline technique," Applied Mathematics and Computation, vol. 181, no. 1, pp. 708$720,2006$.

[5] S. S. Siddiqi and G. Akram, "Septic spline solutions of sixthorder boundary value problems," Journal of Computational and Applied Mathematics, vol. 215, no. 1, pp. 288-301, 2008.

[6] M. A. Noor and S. T. Mohyud-Din, "Homotopy perturbation method for solving sixth-order boundary value problems," Computers \& Mathematics with Applications, vol. 55, no. 12, pp. 2953-2972, 2008. 
[7] A.-M. Wazwaz, "The numerical solution of sixth-order boundary value problems by the modified decomposition method," Applied Mathematics and Computation, vol. 118, no. 2-3, pp. 311325,2001

[8] C. H. Che Hussin and A. Kiliçman, "On the solutions of nonlinear higher-order boundary value problems by using differential transformation method and Adomian decomposition method," Mathematical Problems in Engineering, vol. 2011, Article ID 724927, 19 pages, 2011.

[9] T. E. Simos, "New stable closed Newton-Cotes trigonometrically fitted formulae for long-time integration," Abstract and Applied Analysis, Article ID 182536, 15 pages, 2012.

[10] T. E. Simos, "Optimizing a hybrid two-step method for the numerical solution of the Schrödinger equation and related problems with respect to phase-lag," Journal of Applied Mathematics, Article ID 420387, 17 pages, 2012.

[11] Z. A. Anastassi and T. E. Simos, "A parametric symmetric linear four-step method for the efficient integration of the Schrödinger equation and related oscillatory problems," Journal of Computational and Applied Mathematics, vol. 236, no. 16, pp. 3880-3889, 2012.

[12] G. Akram and H. U. Rehman, "Solution of first order singularly perturbed initial value problem in reproducing kernel Hilbert space," European Journal of Scientific Research, vol. 53, no. 4, pp. 516-523, 2011.

[13] G. Akram and H. U. Rehman, "Numerical solution of eighth order boundary value problems in reproducing kernel space," Numerical Algorithms, vol. 62, no. 3, pp. 527-540, 2013.

[14] G. Akram and H. U. Rehman, "Solution of fifth order boundary value problems in the reproducing kernel space," Middle East Journal of Scientific Research, vol. 10, no. 2, pp. 191-195, 2011.

[15] H. Yao, "New algorithm for the numerical solution of the integro-differential equation with an integral boundary condition," Journal of Mathematical Chemistry, vol. 47, no. 3, pp. 10541067, 2010.

[16] P. K. Pandey, "High order finite difference method for numerical solution of general two- point boundary value problems involving sixth order differential equation," International Journal of Pure and Applied Mathematics, vol. 76, no. 3, pp. 317-326, 2012. 


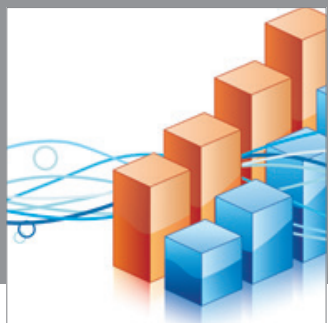

Advances in

Operations Research

mansans

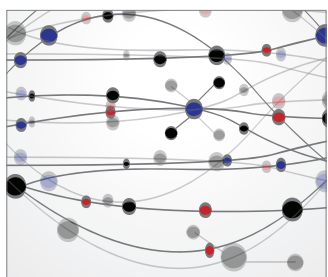

The Scientific World Journal
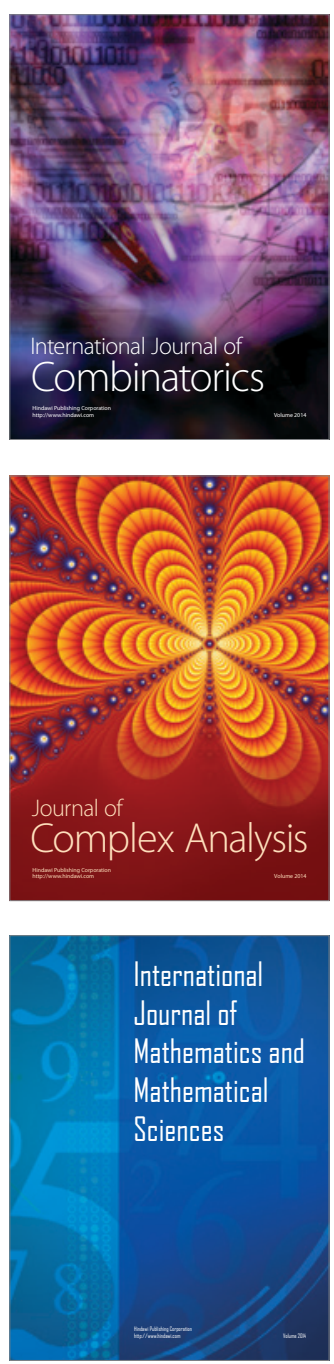
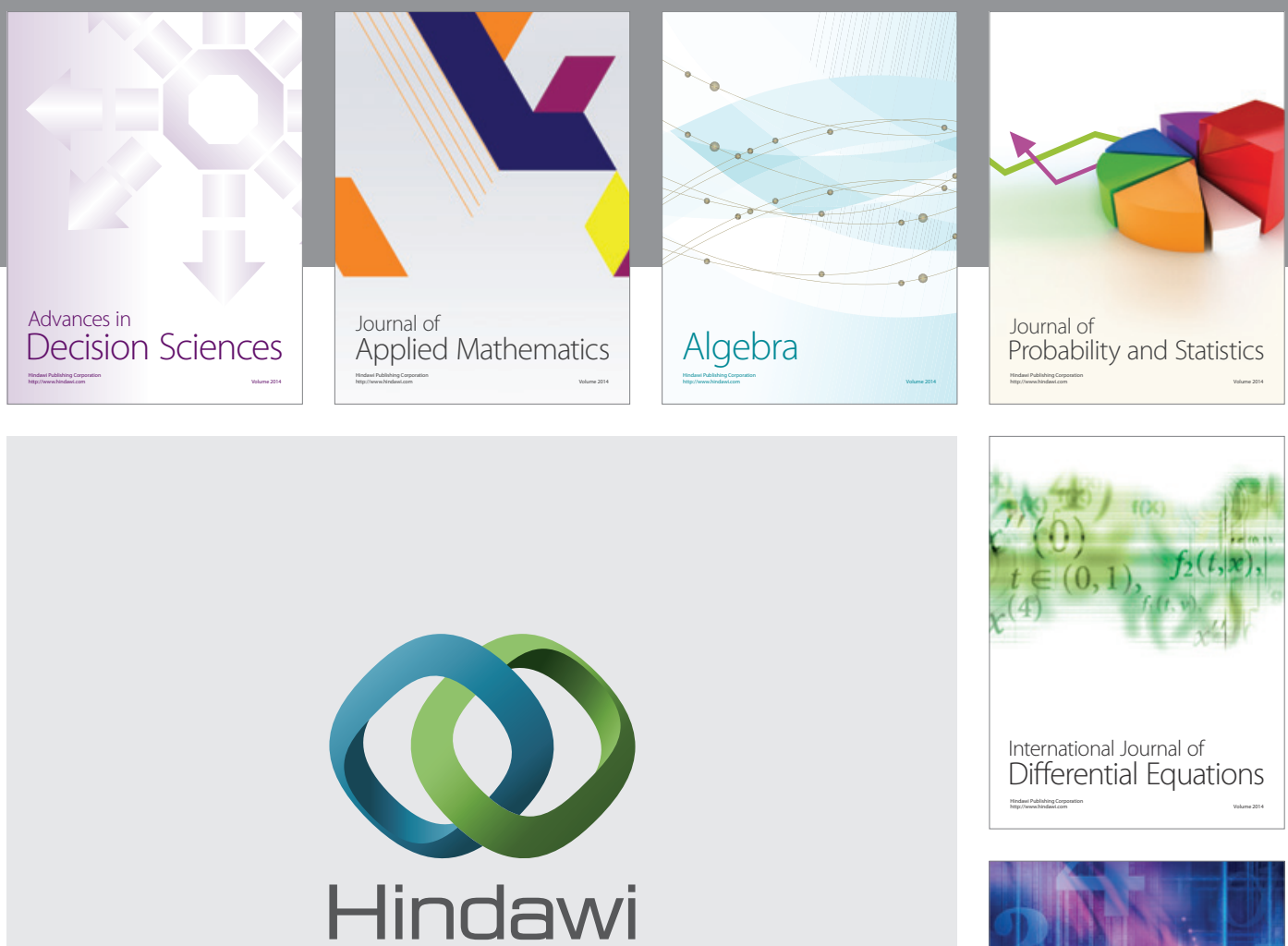

Submit your manuscripts at http://www.hindawi.com
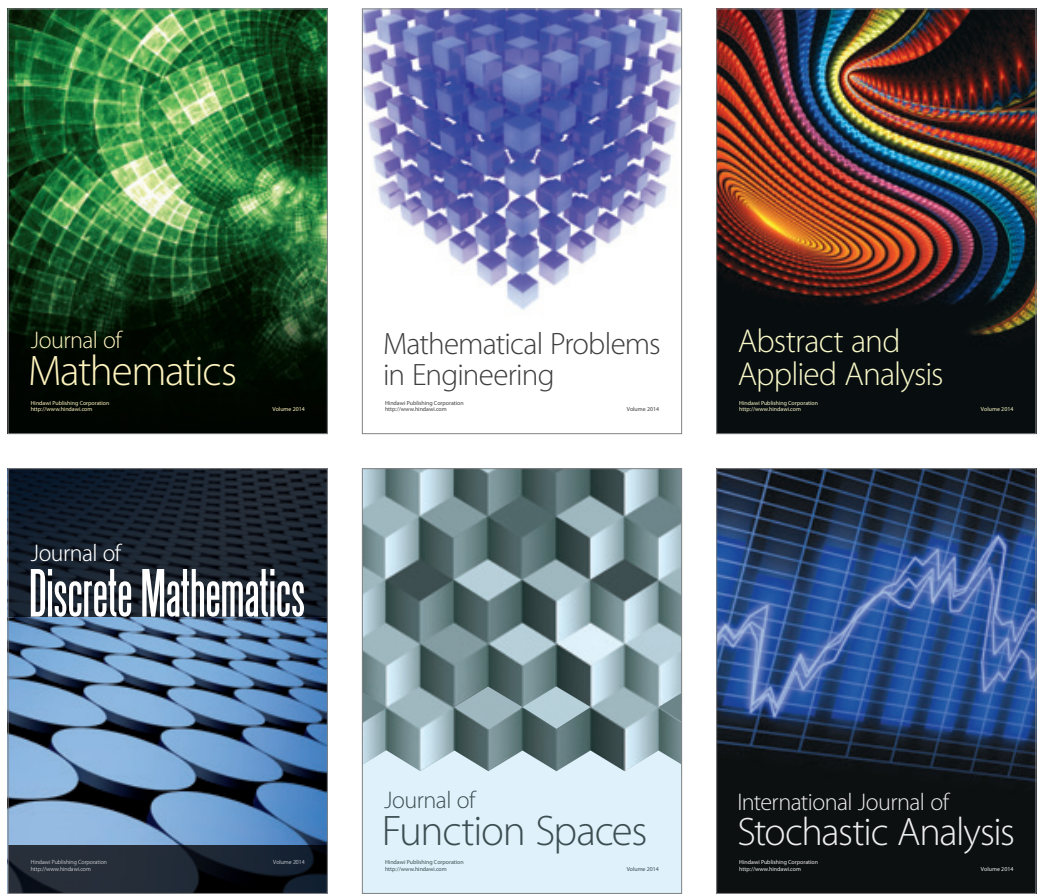

Journal of

Function Spaces

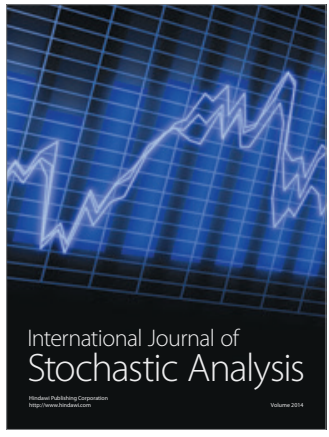

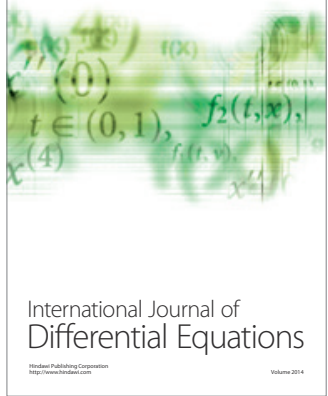
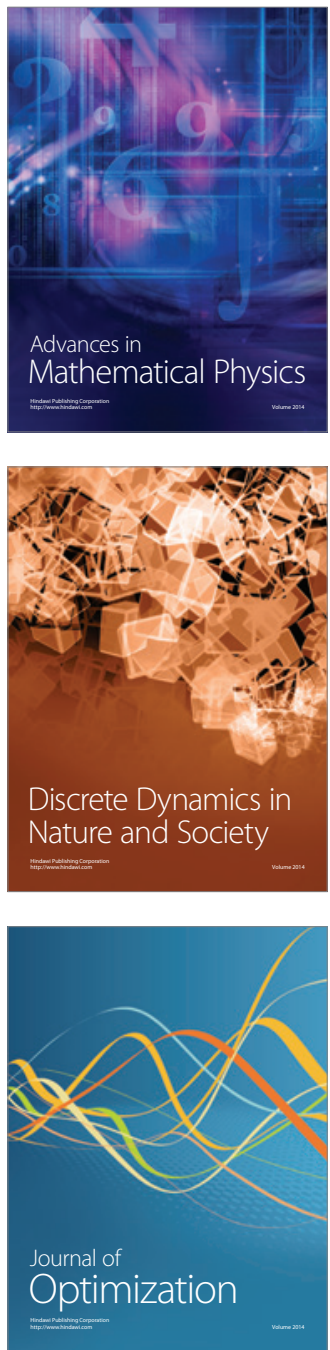